\title{
A PROPÓSITO DE UN CASO DE CONVERGENCIA GRAMATICAL POR CAUSACIÓN MÚLTIPLE EN EL ÁREA DE INFLUENCIA LINGÜÍSTICA CATALANA. ANÁLISIS SOCIOLINGÜÍSTICO
}

\author{
José Luis Blas Arroyo* \\ Universidad Jaime I
}

\begin{abstract}
The paper represents a study of a Spanish syntactic phenomenon in the Catalonian speech communities as a case of grammatical convergence between both languages. To the author, the origin of the concordance feature between the Spanish verb haber and the noun in normative impersonal sentences may be internally induced, but his great social extension is only explicable by linguistic contact influence. The presence of this feature in other Spanish varieties doesn't mean that the accomplishment of the linguistic change has the same explicative factors in all the speech communities. Because of this epistemological principle, the author makes a cross dialectal analysis of the linguistic variable in the Spanish and the Catalan languages. In the empirical work he treats some sociological factors as mother tongue, dominant languages or origin of the speakers that reveals the importance of linguistic contact in the realization of the concordance. Finally, he makes some consideration about the problems that has had the interference concept in linguistics and sociolinguistics in last times, as well as about the implicit-and erroneousidea that the contact induced changes are only unidirectional (from the more to the less prestigious languages).
\end{abstract}

RESUMEN: El trabajo representa el análisis teórico y empírico de un fenómeno sintáctico característico del español en las comunidades de habla del área lingüística catalana. En éstas, la concordancia entre el verbo haber y el núcleo del sintagma nominal subsiguiente (habían flores), en oraciones que la normativa del español considera como impersonales, puede ser explicada como un fenómeno de causación múltiple, para el que la convergencia gramatical entre español y catalán tiene una importancia decisiva. La presencia del mismo rasgo sintáctico en otras variedades del español no impide dicha caracterización ya que causas diversas pueden llevar ocasionalmente a idénticos resultados en áreas alejadas geográficamente. Tras un análisis comparativo del fenómeno en ambas lenguas, el autor comenta las principales conclusiones de un estudio empírico llevado a cabo en una comunidad de habla valenciana, en el que variables sociológicas como la lengua nativa y/ habitual o el origen de los hablantes se han tenido especialmente en cuenta, junto a otras habituales en esta clase de trabajos (sexo, edad, nivel de estudios). Los datos confirman la hipótesis de que la concordancia es un fenómeno ampliamente extendido en la sociedad valenciana, pero especialmente entre los grupos sociales más autóctonos por adscripción lingüística u origen. Asimismo, en el trabajo se realizan algunas reflexiones acerca de ciertos problemas que han lastrado el estudio de la interferencia lingüística en los últimos tiempos, en particular la idea -errónea-, de que aquélla sólo actúa en la dirección que va desde la lenguas más prestigiosa o dominante hacia las demás.

\footnotetext{
* Prof. del Dpto. de Filología. Universitat Jaume I, Castelló.
} 


\section{Introducción}

Pese al éxito cosechado a raíz de la obra de Weinreich (1953), el concepto de interferencia ha vivido en los últimos años horas muy bajas, y sólo aisladamente algunos autores han levantado la voz desde diferentes disciplinas, como la sociolingüística o la lingüística histórica, para rehabilitarlo (Mougeon y Beniak 1991; Thomason y Kaufman 1988). Este descrédito tiene diferentes causas. Para empezar y como han subrayado Mougeon y Beniak (1991: 181), a la interferencia le ha pasado como a tantos otros conceptos científicos, que tras un periodo de esplendor han visto cómo eran sometidos a la críticas más acerbas. A ello ha contribuido, sin duda, el abuso con que se ha echado mano con frecuencia del contacto lingüístico para explicar cualquier cambio en la evolución de las lenguas. Explicaciones exageradas, cuando no simplemente erróneas, que denotaban asimismo fallos graves en el aparato metodológico de las investigaciones.

Al panorama anterior hay que añadir otras deficiencias, cometidas incluso cuando el propio concepto de interferencia gozaba de buena salud. Así, por ejemplo, frente al éxito de los estudios sobre las consecuencias de la influencia interlingüística en algunos niveles del análisis como el fónico o el léxico, la atención dedicada al estudio de la interferencia gramatical ha sido mucho menor, entre otras razones por la creencia -errónea- de que los subsistemas morfológico y sintáctico son inmunes al préstamo (cf. Payrató 1985; Thomason y Kaufman 1988; Blas 1991). Por otro lado, el interés por la variación y el cambio linguiístico en los que han podido intervenir tanto factores internos como influencias externas -lo que aquí denominamos causación múltiple- ha sido muy limitado y sólo recientemente se ha reivindicado como un factor digno de ser considerado en la evolución de las lenguas.

A nuestro juicio, otro de los aspectos más olvidados, incluso por aquellos que reivindican el papel de la interferencia en la evolución de los sistemas lingüísticos, es el relativo a la dirección de ésta. Existe un consenso ampliamente difundido, unas veces explícita y otras implícitamente, según el cual la dirección de la interferencia sólo se da en el sentido que va desde la lengua con mayor prestigio hacia la lengua con menos estatus social. El que viejos conceptos procedentes de la lingüística histórica, como el de sustrato, vengan a la mente en este caso no invalida la afirmación anterior, ya que el recurso a éste y otros similares ha sido objeto con frecuencia de un uso superficial e inconsecuente.

Coincidimos con aquellos autores que como Beniak o Mougeon (1991), apuestan por la rehabilitación de la interferencia con criterios de responsabilidad científica, pero por nuestra parte añadiríamos la necesidad de que aquélla alcance todas las facetas que el contacto de lenguas puede adquirir. Sólo el conocimiento profundo de la realidad sociolinguiística de cada comunidad de habla puede ayudarnos a interpretar cabalmente el sentido de la influencia interlingüística (cf. Thomason y Kaufman 
1988), por lo que las predicciones a partir de factores excesivamente generalizantes como el prestigio y otros similares se nos antojan inútiles.

El principal objetivo del presente trabajo es el análisis de un fenómeno sintáctico característico del español en el área lingüística catalana. Se trata de la concordancia entre el verbo y el sintagma nominal subsiguiente en frases como:

(1) Habian flores en el jardín

(2) Hubieron fiestas en el pueblo

(3) Han habido muchos disturbios en la calle

El fenómeno de la concordancia entre el sintagma nominal y el verbo haber en este tipo de oraciones, que la norma caracteriza como impersonales, no es nuevo en la historia del español, ni tampoco privativo de las comunidades de habla mencionadas. Sin embargo, y al menos en el español peninsular, existen diferencias de grado importantes entre la extensión social del fenómeno en las regiones en las que español y catalán conviven y el resto. Hay que subrayar que el mismo rasgo sintáctico es sumamente característico del catalán, y aunque tampoco normativo como en español, se ha llegado a plantear como variante categórica en algunos lugares.

La hipótesis que formulo y sobre la que gira el cuerpo fundamental del trabajo es que dicho fenómeno constituye un ejemplo de convergencia gramatical entre dos lenguas romances como el español y el catalán, al que se ha llegado al cabo de numerosas generaciones de intenso contacto. El hecho de que tanto en una lengua como en otra pueda ser fácilmente explicado a partir de factores puramente internos, no debe impedir la consideración de la influencia interlingüística como un hecho determinante en el éxito y la extensión social del mismo. Es a esta conjunción de factores internos y externos, como la interferencia lingüística, a la que denominamos causación múltiple (cf. Thomason y Kaufman 1988; Germán de Granda 1991; Silva Corvalán 1994).

Para poner a prueba esta hipótesis hemos realizado una investigación empírica en una de esas comunidades de habla. A través de ella, y en especial mediante la consideración de algunas variables sociológicas, como la adscripción lingüística o el origen de los informantes, esperamos confirmar algunas diferencias que intuimos entre la actuación sociolingüística de estas sociedades bilingües y otras áreas del español peninsular. La necesidad de esta comparación interdialectal entre diversas variantes del español se impone, si quiere darse cuenta de las diferencias que un mismo fenómeno puede ofrecer en comunidades diferentes de una misma lengua. En este caso, sin embargo, la comparación no puede realizarse entre estudios empíricos de la misma entidad ya que, al menos hasta donde llega nuestro conocimiento, no existen análisis similares para otras zonas de la lengua española. Existen, no obstante, referencias bibliográficas que dan cuenta de la extensión social del fenómeno en otras comunidades. Como contrapartida, en esta investigación evaluaremos con especial cuidado las eventuales diferencias de comportamiento sociolingüístico que se aprecien entre dife- 
rentes grupos sociales, como los hablantes bilingües y monolingües, o las existentes entre los individuos oriundos de la comunidad de habla y quienes se han incorporado como inmigrantes en un momento posterior de sus vidas.

Pero antes de pasar al estudio de los datos arrojados por la investigación empírica, analizaremos con más detalle alguno de los aspectos esbozados en esta introducción sobre las deficiencias teóricas y metodológicas de la interferencia interlingüística. A nuestro juicio, ello puede contribuir a que el lector comprenda mejor los extremos de la investigación. A tal efecto dedicamos el siguiente epígrafe al análisis de los problemas que plantea el estudio de conceptos básicos en el trabajo como la causación múltiple o la convergencia gramatical. Por último, y con el fin de situar mejor el alcance estructural y sociolingüístico del fenómeno, dedicaremos también un apartado a la descripción del mismo en español y catalán.

\section{La causación múltiple en la variación y la evolución de las lenguas}

Sólo recientemente se ha admitido la causación múltiple en la configuración de la variación y el cambio linguiísticos, esto es, la intervención simultánea de factores internos al propio sistema y de factores externos, como la influencia de una segunda lengua, que pueden actuar como catalizadores de los primeros. Con todo, ya Vogt (1954: 372) apuntó que las diferencias entre dialectos alejados geográfica y culturalmente podrían tener en la interferencia lingüística una explicación plausible. De esta manera, si bien las posibilidades de innovación estarían ya latentes en la propia lengua, las diferencias entre unas variedades y otras podrían deberse al contacto lingüístico.

La necesidad de un análisis interdialectal para determinar el grado de influencia posible de una segunda lengua ha sido advertido recientemente por diversos autores. Para Mougeon y Beniak (1991: 181ss), por ejemplo, cuando la ambigüedad interferencia versus desarrollo interno desemboca en una simplificación en la evolución de la lengua, es difícil determinar si una de tales causas hipotéticas es más relevante que la otra1. Por el contrario, cuando el resultado de la variación o el cambio es una complicación estructural, resulta verosímil colocar en primer término la influencia interlingüística como factor explicativo esencial (véase también Thomason 1986).

\footnotetext{
1. A propósito del uso de la preposición sur en algunas modalidades del francés canadiense para expresar circunstancias locativas (sur la television en lugar de à la television ), estos autores han demostrado que el fenómeno se produce en el estado de Ontario, un área de contacto en la que la presión del inglés es muy fuerte, pero no en la vecina Quebec. Sin embargo, el rasgo gramatical se documenta también en variedades del francés europeo, es decir, en dialectos en los que el contacto de lenguas no tiene lugar, lo cual hace pensar que se trata también de un hecho gramatical que tiene una justificación interna: "It seems to us that linguists ought to avoid forcing an interpretation for the structural origin of these changes and openly acknowledge the possibility of multiple causation" (véase Thomason y Kaufman 1988 para una opinión similar)
} 
A nuestro juicio, sin embargo, no existen razones para suponer que esta regla tiene carácter universal. Es lógica su aplicación para los hablantes cuyo empleo de segundas lenguas está restringido socialmente ${ }^{2}$. En tales casos, ciertamente, la simplificación puede ser un simple mecanismo estructural al que se accede por la concurrencia de los factores que dan lugar al uso de una lengua restringida, sin necesidad de recurrir a la interferencia. Ahora bien, en las comunidades de habla donde ello no ocurre, es decir, donde el acceso a la segunda lengua no se encuentra limitado por ningún tipo de restricción social, cultural o interaccional, los procesos de simplificación pueden verse favorecidos por el contacto, situándose la interferencia en cabeza de los factores explicativos de la variación. Como intentaremos demostrar más adelante, esto es lo que, a nuestro juicio, ocurre con la variable sintáctica objeto de estudio en este trabajo. La definitiva integración social de la concordancia en oraciones impersonales con haber en las comunidades de habla del área lingüística catalana es, sin duda, el desenlace de un proceso de simplificación en español -y en catalán-, pero a ello ha contribuido decisivamente la existencia de un fenómeno similar en la segunda lengua, en este caso, por cierto, la lengua minoritaria desde un punto de vista sociocultural.

La simplificación gramatical es, efectivamente, uno de los tipos de cambio que con más frecuencia se han identificado en las situaciones de contacto. Puede ser definida como la emergencia de innovaciones que representan alternativas más simples, en el sentido de más regulares, más transparentes, que las variantes normativas (a su vez más opacas e irregulares) (cf. Trudgill 1983: Cap. 6; Mougeon y Beniak 1991: 217). Ahora bien, no siempre el concepto de simplificación se ha utilizado en este sentido. Como ha recordado Fishman (1982: 58), las nociones de simplicidad y simplificación se encuentran entre los conceptos más ambiguos y escurridizos que puede haber en la caracterización del lenguaje. Por su parte Silva Corvalán (1994: 4), otra de las autoras que ha hecho uso del término recientemente, define la simplificación como el uso más frecuente de una forma $X$ en un contexto determinado en detrimento de otra forma, Z, relacionada con la anterior, donde ambas, $\mathrm{X}$ y Z, son variantes que existen en la lengua previamente al proceso descrito.

A nuestro juicio, la simplificación supone un poco de todo lo anterior. Por un lado, se trata de formas latentes en la propia estructura de la lengua, que ocasionalmente han podido aparecer en épocas diversas de su evolución en algunas variedades dialectales y que lógicamente, tras un proceso de cambio han podido triunfar socialmente sobre las variantes con las que competían. Por otro, son también unidades lingüísticas que vienen a limitar o a eliminar paradigmas irregulares en determinados subsistemas de la lengua y como consecuencia de ello, a instaurar un nuevo orden más transparente.

2. Así ocurre, según describen Mougeon y Beniak en la obra reseñada, con el francés en la región de Ontario. 
Ahora bien, los intentos por delimitar claramente este proceso de cambio frente a otras consecuencias del contacto pueden resultar más complicados de lo que algunos están dispuestos a admitir. Por ejemplo, Silva Corvalán (1994; 1995) subraya la división anterior como una necesidad imperiosa y advierte sobre los excesos en la atribución a la interferencia de responsabilidades en los cambios. La lingüista chilena distingue a este propósito entre simplificación, interferencia -que ella denomina transferencia (transfer) ${ }^{3}$-, convergencia y análisis ${ }^{4}$. Para ella, la transferencia puede conducir a la convergencia gramatical, aunque no tiene por qué ser la única causa. Por convergencia, Silva Corvalán entiende la consecución de un grado de proximidad estructural en un aspecto determinado de la gramática entre dos o más lenguas (cf. Gumperz y Wilson 1971). Aunque la convergencia puede ser el resultado de cambios preexistentes motivados por causas internas en la lengua, la investigadora americana admite que ésta puede verse acelerada por el contacto "rather than as a consequence of direct interlingual influence" (Silva Corvalán 1986: 593).

Desde este punto de vista, en la convergencia gramatical habría que distinguir dos etapas, el origen del cambio -debido a factores puramente internos- y la difusión del mismo, que el contacto interlingüístico podría favorecer, pero sin que pueda verse en él la causa fundamental. La siguiente cita condensa el pensamiento central de la autora a este respecto:

I favour a slightly different hypothesis: that the structure of the languages involved, to a large extent constrained by cognitive and interactional processes, governs the introduction and diffusion of innovative elements in the linguistic systems; the sociolinguistic history of the speakers is the primary determinant of the language direction and the degree of diffusion of the innovations as well as of the more distant (in terms of time-span) linguistic outcome of language contact (i.e. after several generations of normal language transmission; cf. Thomason and Kaufman I988). This hypothesis accounts for the changes attested in numerous situations of language maintenance and/or shift involving normal transmission across generations.

3. Sobre la inadecuación de este término para dar cuenta de los procesos interferenciales ya que sólo contempla una de las posibilidades de la influencia interlingüística -la migración de elementos lingüísticos de una lengua a otra- desatendiendo otras más complejas, véanse Payrató (1985) y Blas (1991). De hecho, la propia autora advierte implícitamente estos problemas nominativos cuando explica ciertos fenómenos claramente interferenciales que, sin embargo, no suponen una novedad estructural en la lengua meta. Ello se debe en gran medida a la transparencia del término transferencia, que como hemos dicho, deja escasos resquicios para la explicación de otras consecuencias lingüísticas del contacto.

4. La autora entiende por análisis: "the process which underlies either the preferential use or the creation of analytical or periphrastic constructions as opposed to synthetic ones. This accounts, for instance, for the much more frequent use of the periphrastic future and conditional forms (ir a 'go to' + Inf.) rather than the corresponding synthetic ones (-rá, -ría) in all varieties of Spanish...” (Silva Corvalán 1994: 5). 
Es difícil estar en contra de una formulación como la anterior, en la que factores estructurales y externos se dan cita para la configuración de los cambios. Ahora bien, tanto en ésta como en otras formulaciones similares, se aprecia a nuestro juicio, una separación excesivamente tajante entre la responsabilidad de ambos factores en la variación y el cambio lingüísticos. Decir que el cambio puede explicarse a partir de factores puramente internos, que han estado latentes en la evolución de la lengua, no es exactamente igual que admitir que ésta es la causa primera de dichos fenómenos de convergencia y que el contacto sólo vendría, en el mejor de los casos, a acelerar el proceso. Y eso es precisamente -al menos, así nos lo parece- lo que parece derivarse de citas como la anterior. A nuestro juicio, por el contrario, lo que en una comunidad de habla puede ser así no tiene por qué reproducirse de la misma manera en otras. El resultado final podría ser idéntico, pero no la combinación de causas que se han dado cita en su configuración. Por tanto, factores internos y contacto interlingüístico desempeñan funciones cuya prelación es difícil de establecer, labor para la que, en todo caso, es necesario el auxilio de la sociolingüística. El hecho de encontrar una explicación al cambio por la vía de acudir simplemente a las posibilidades internas de la lengua no significa que ésta sea la única, ni siquiera la principal. Por ello mismo, las fronteras entre procesos como el de simplificación o el de convergencia se nos antojan frágiles en demasiadas ocasiones.

En suma, que en un comunidad de habla bilingüe, en la que la intensidad del contacto interlingüístico es elevada, se rechace la influencia de éste como factor determinante en la variación, por el simple hecho de que los fenómenos son fácilmente interpretables a partir de causas meramente estructurales, nos parece una interpretación errónea de los hechos lingüísticos.

\section{La variable lingüística}

Como avanzamos al comienzo de este trabajo, consideramos que la concordancia sistemática entre el verbo y el núcleo del sintagma nominal que le sigue en oraciones que, como (3) y (4), han sido caracterizadas -y prescritas- tradicionalmente como impersonales, constituye uno de los rasgos más definidores del español hablado en las comunidades del área lingüística catalana:

\section{(3) Habian muchas flores en aquel jardín \\ (4) Están haciendo unos días muy buenos.}

El fenómeno afecta, como veremos, a verbos como haber o hacer en usos gramaticalizados, si bien en este trabajo sólo nos ocuparemos del primero, por ser el que presenta un perfil más nítido, tanto por su elevada frecuencia en términos absolutos -v. gr. aparición en el discurso de esta clase de oraciones con dicho verbo en detrimento 
de las frases con haber- como en términos relativos -v. gr. en relación a la otra variante, es decir, la construcción normativa en la que no se practica la concordancia-.

Pensamos que en estas comunidades de habla el fenómeno puede ser interpretado como un caso de convergencia gramatical al que contribuyen no sólo la simplificación interna de un paradigma poco habitual, como el que representa la ausencia de concordancia en esos casos, sino también la influencia social que ejerce idéntico fenómeno en la mayoría de las variedades del catalán:

(5) Hi avien moltes flors...

(6) Hi están fen uns dies molt bons.

El rasgo de la concordancia contradice la consideración de estas oraciones como impersonales, tal y como han sido clasificadas por la normativa actual. No obstante, no hay unanimidad entre los lingüistas a la hora de decidir si realmente el sintagma que sigue al verbo es sujeto o complemento directo. Como ha mostrado García Yebra (1983: 33-71), las discrepancias entre los gramáticos constituyen a este respecto la norma. Por lo que se refiere al español, sin embargo, la mayoría de los autores se decantan por la consideración de "haber" como transitivo y, por lo tanto, como impersonales a las construcciones donde este verbo aparece. Así es, por ejemplo, para Bello (1945: 225), la Gramática de la Real Academia (1931: 3.5.7.c), Gili Gaya (1961: 78), Manuel Seco (1961: 215), Hernández (1984: 139), Alcina y Blecua (1975: 890), etc. Manuel Seco (1961/86: 215), en su Diccionario de dudas y dificultades de la lengua española, condensa bien esta doctrina normativa en la siguiente cita:

Haber 2. Transitivo impersonal con el significado de "encontrarse". En este uso, el indicativo presente tiene la forma especial hay: hay habitaciones libres. No es normal el empleo de haber impersonal con un sustantivo determinado, como ocurre en este ejemplo de Galdós: "hay en Madrid los grandes ópticos" (Torquemada en la Cruz); y en éste de Aranguren: 'Allende presente, mundo real Sobre él hay el otro mundo': no ya real, sino realisimus" (Crítica y meditación). El verbo haber conserva en estas construcciones un resto del sentido antiguo "tener", pero su sujeto es indeterminado; el sustantivo que lo acompaña es complemento directo .

García Yebra (1983) resume así las principales razones que se han esgrimido para considerar complemento directo y no sujeto a los sintagmas en cuestión:

1) El sujeto de hay es vago e indeterminado, y su idea "se ofrece de un modo oscuro y fugaz al entendimiento, concordando con el verbo en tercera persona de singular (Bello, 1951: 225). El sustantivo regido por hay es complemento directo de este verbo. "Por eso construimos el verbo en singular cuando decimos hubo toros (...) y no hubieron toros" (GRAE, 1931, n. ${ }^{\circ} 284$ c). Dicho de otro modo: un sujeto plural tendría que llevar el verbo en plural; luego toros no puede ser sujeto de hay. Tal sería el 
corolario de la siguiente descripción de sujeto: "Frente a la definición lógico-semántica que daba la gramática tradicional, se postula la distinción del sujeto por el hecho de que es el único elemento nominal que cambia su marca de número con el verbo con el cual concuerda" (Alcina-Blecua, 1975: 853).

2) "Que la cosa cuya existencia se significa (mediante la tercera persona del singular de haber) está en acusativo, lo prueba la necesidad del caso complementario de acusativo cuando la representamos con el pronombre él (...) Si el impersonal haber significara de suyo existir, sería la mayor de todas las anomalías poner las cosas existentes en acusativo" (Bello, supra, 1.1). Por eso, "cuando se nos pregunta: ¿hubo heladas? contestamos: las hubo, empleando la forma del pronombre que sabemos es de acusativo" (GRAE, supra, 1.3).

Sin embargo, García Yebra (1983) se alía junto a quienes ven en el SN un sujeto y no un objeto directo. Para el académico español, los argumentos anteriores no sólo no son definitivos sino que pueden ser contrarrestados por otros de mayor peso científico. Por ejemplo, al hecho de que el verbo concierte en número con el sustantivo y de ahí que, en hubo toros, esta última palabra no pueda ser sino complemento, García Yebra recuerda que la discordancia es un fenómeno común en las lenguas romances, y entre ellas el español. Además de los casos en que el núcleo del sujeto posee un rasgo semántico de colectividad ("La multitud votaron guerrear") o aquéllos en los que tanto el hablante como el interlocutor están incluidos en el ámbito de referencia del sujeto (los estudiantes pensamos/pensáis que no tienen razón) (vid. Moreno Cabrera 1991), existen en la lengua otros ejemplos de discordancia numérica como la que se produce con los llamados verbos "anquilosados". Así ocurre, por ejemplo, con la forma "he" cuyo origen adverbial y no verbal ya señaló Corominas (1954; v. 11l, 33435) (dicho elemento se sintió desde antiguo como una segunda persona del imperativo de "haber" con valor transitivo).

Por otro lado, la conmutación pronominal por una forma de acusativo en:

\section{(7) ¿Has visto hombres con cinco piernas? No, no los he visto}

que debería ser prueba suficiente para la consideración como objeto directo del sintagma, podría explicarse simplemente como un resto fosilizado del uso primitivo de haber, fundamentalmente transitivo, y equivalente al actual tener.

A partir de estos y otros argumentos que no desarrollaremos más, García Yebra (1983) concluye que:

La norma lingüística del español rechaza todavía esa concordancia, que los hablantes lingüísticamente cultos consideran vulgarismo. Pero también la gran mayoría, por no decir la totalidad, de esos hablantes conocedores y practicantes de la norma lingüística sienten como sujeto - aunque no concierte en número con el verbo- el sustantivo o elemento sustantivado de referencia. Sólo entre 
los gramáticos más atentos a la primitiva significación de haber que a su evolución sintáctica hay quienes sostienen la opinión contraria (García Yebra, 1983: 69).

Puede que sea excesivo suponer que los hablantes tienen éste u otro sentimiento respecto a una función u otra, independientemente de que practiquen o no la concordancia. Pero parece indudable que cuando ésta se realiza, la consideración de objeto directo para el sintagma nominal es sólo un academicismo que no se corresponde con la competencia lingüística de los hablantes.

Aunque no pretendemos adherirnos aquí a ninguna de las dos posiciones teóricas, lo cierto es que la estructura normativa de estas oraciones impersonales, con el verbo siempre en la tercera persona del singular, supone una engorrosa excepción para el sistema sintáctico del español, lo que permitiría justificar la práctica de la concordancia en diversas variedades. Así, por ejemplo, dos lingüistas del siglo pasado como Salvà (1831) y Bello (1874), en sendas obras sobre el uso del castellano en la época, advertían ya a sus coetáneos sobre el "vicio" de la concordancia. Ya en nuestro tiempo, diversos autores han realizado también algunas referencias a la extensión progresiva en el mundo hispánico de la concordancia entre el verbo y el sustantivo. Para Fernández Ramírez (1986), por ejemplo, el aislamiento en que viven, sin apoyo en otras formas semejantes, las terceras personas del singular del transitivo "haber", de uso tan frecuente en español, puede explicar la fuerte tendencia que se observa en el habla descuidada y en el habla popular (el subrayado es nuestro) a consentir la relación verbo-complemento en la relación verbo-sujeto, haciendo plural el verbo cuando el complemento es también plural (Fernández Ramírez, 1986: 132-33; véase también Maldonado de Guevara 1980: 30-31).

Sin lugar a dudas, la mayor cantidad de alusiones respecto a la extensión del fenómeno remiten al español de América, lo cual contrasta con las referencias mucho más escasas disponibles para la península. No es casual a este respecto que García Yebra mencione que la concordancia se produce "sobre todo entre los hispanoamericanos" (pág. 68). Asimismo, lingüistas de este continente lo vienen documentando desde hace bastante tiempo en el español del otro lado del Atlántico. Como ya hemos indicado, Bello opinaba que la consideración del verbo "haber" como personal era, por ejemplo, "un vicio casi universal en Chile" (citado en Fernández, 1986: 132). Kany, por su parte, añade algunos comentarios interesantes de carácter sociolingüístico y asegura el uso muy común de la concordancia en la lengua escrita y hablada de toda América, aunque dice hallarse especialmente extendida por Argentina, Chile y América Central (Kany, 1945: 212-15). Según este autor, por último, son muy pocas las regiones que se libran de este uso popular, "y en muchas partes, las personas cultas y los mejores escritores emplean la forma popular junto a la forma correcta" (Kany, 1945: 215).

Las referencias bibliográficas más recientes parecen confirmar el éxito del fenómeno en el español de América, aunque quizá no con la contundencia con que lo suponía el lingüista norteamericano hace cincuenta años. A este respecto, por ejemplo, 
hemos entresacado algunos datos de una obra reciente donde se realiza un repaso sucinto, pero bastante completo, sobre los principales rasgos del español de diversas áreas dialectales americanas (Hernández 1992). En ella, por ejemplo, J. J. Montes (1992: 534-5) subraya que "la concordancia verbo-sustantivo (es) norma casi única" en Colombia "y no sólo en la lengua hablada". Y añade a continuación: "tengo muchos ejemplos de literatos (García Márquez, historiadores, etc.). Incluso en mi habla natal (Manzanares, Caldas) es usual el plural en presente (haen hain)". Por su parte Bentivoglio y Sedano (1992: 791) apuntan que, pese a la prescripción normativista en contra "los venezolanos suelen hacerlo concordar [el verbo haber] con el sustantivo que lo acompaña, y si éste es plural, haber también está en plural". Además añaden- "la pluralización de haber se da -con excepción de hay- en todos los modos y tiempos (habian, habrán, hubieron, hayan...), pero la frecuencia de uso varía según diversos factores. De las distintas formas que admiten pluralización, la de uso más restringido, quizá por razones tanto fonéticas como sociolingüísticas, es hubieron. Esta situación podría cambiar en el futuro pues hubieron se está oyendo cada vez con más frecuencia entre los jóvenes de todos los niveles". Este último dato sugiere que el fenómeno que nos ocupa no está definitivamente consolidado, sino que continúa en expansión.

Otras referencias sitúan el rasgo de la concordancia en otras variedades dialectales y sociolectales del español de América. Así, Caravedo (1992: 726) apunta que constituye la norma entre "todas las clases sociales" en el Perú costeño y añade como dato interesante que "además es percibido como correcto". No dice nada, sin embargo, respecto a la extensión en otras áreas, como el español andino o en el amazónico. Quien sí documenta el fenómeno en el castellano andino de Bolivia es Mendoza (1992: 458) a partir de un pequeño trabajo empírico, aunque no añade ningún otra caracterización. Igualmente documentado está por Quilis (1992: 604) en Ecuador, si bien este tipo de oraciones en opinión del autor "ofrecen un comportamiento muy heterogéneo, tanto en las localidades, como en los informantes". Y Moreno de Alba (1992: 640), recuerda también, aunque sin más precisiones, que en México "es frecuente el empleo personalizado del verbo haber, que deja de sentirse como transitivo sin sujeto ("había sorpresas") y se le hace concordar con un supuesto sujeto ("habían sorpresas"). Curiosamente, por último, no encontramos referencias sobre el fenómeno de la concordancia en el español hablado en Argentina, Chile o Uruguay, en los que Kany creía ver el fenómeno especialmente extendido, si bien es posible que tales ausencias se deban sobre todo a las restricciones impuestas por el propio tipo de trabajo del que están extraídas.

Por lo que se refiere al español de España, que es el que aquí nos interesa sobre todo para poner a prueba nuestras hipótesis, las referencias son más escasas e imprecisas. Para Alcina y Blecua (1975: 890), el habla culta en Castilla y en casi toda la península mantiene el singular; sin embargo, la lengua hablada y aun la escrita, en periódicos y traducciones, emplea el plural, sin precisar más sobre la posible estratifi- 
cación sociolinguística y dialectal del fenómeno. Por su parte, Lapesa (1986) considera que el fenómeno está en pleno apogeo, si bien lo circunscribe también sociolectalmente a dominios de habla populares y vulgares. Recuérdense igualmente a este respecto las impresiones de Fernández Ramírez anteriormente reseñadas.

Manuel Seco (1961/81: 216), por su parte, subraya que en España el fenómeno se produce principalmente en "el área del catalán y en hablantes procedentes de ellas". Una distribución dialectal que es confirmada por Maldonado de Guevara con algunos añadidos. Ardiente defensor de la normativa que impone la no concordancia, este autor califica estas construcciones como "flagrantemente vulgares" y añade que está muy difundida por el dominio lingüístico del español, aunque "tiene un carácter vulgar, y es muy sintomática de un bajo nivel cultural". Apoyándose en su propia experiencia, Maldonado de Guevara establece una distribución dialectal y sociolectal del fenómeno en España que nos interesa especialmente. Establece una diferencia clara entre la difusión de la concordancia en regiones como la parte oriental de Aragón, la Mancha oriental, Murcia y Andalucía oriental por un $1 \mathrm{lado}^{5}$, y las comunidades de habla del área de influencia lingüística catalana, por otro. En las primeras, el fenómeno tiene un carácter marcadamente vulgar, a juicio del lingüista español, y las clases cultas son conscientes de ello. Por el contrario, en las comunidades del dominio catalán las oraciones en las que se practica la concordancia

...son un bien mostrenco de toda a comunidad, independientemente del estrato sociocultural y del grado de instrucción de cada persona. En Cataluña, Baleares y Reino de Valencia, todos, absolutamente todos los hablantes, cometen sistemáticamente esta incorrección, y no tienen conciencia, por cultos que sean, de que están cometiendo un grave pecado gramatical. Esto se puede comprobar fácilmente; basta para ello escuchar a cualquiera de los locutores de la Radio o de la Televisión de las emisoras catalanas, valencianas y baleares (el subrayado es nuestro)

A nuestro juicio también, la concordancia entre el verbo "haber" y el núcleo del sintagma nominal no tiene idéntica extensión por toda la península. En ausencia de estudios empíricos que confirmen o desmientan esta hipótesis, y guiados de momento sólo por nuestra experiencia y por referencias como las anteriores, nos parece, sin embargo, que el fenómeno de la concordancia tiene una enorme vitalidad en las regiones del área de influencia catalana. Una vitalidad que no se detiene en las capas más populares de la población, como puede ocurrir en otros territorios, sino que se ha infiltrado poderosamente en todos los estratos de la sociedad, y cuyo reflejo aparece tanto en la lengua hablada como, también—frecuentemente—, en la escrita 6 .

5. ¿Es casualidad que se trate de las regiones más cercanas geográficamente al área lingüística catalana o incluso donde alguna modalidad de esa lengua se ha hablado o todavía se habla? 
Desde nuestro punto de vista, al hecho estructural favorecedor de la concordancia entre el verbo y el SN, hay que sumar, en el contexto social del bilingüismo catalán-español, el refuerzo importante de un fenómeno idéntico en los hábitos lingüísticos de los catalanohablantes. Y es que, a pesar de las reconvenciones normativas de los gramáticos de esta lengua, la concordancia es un rasgo extraordinariamente habitual también en el catalán de estas comunidades. Badia, por ejemplo, en su Gramática catalana alude a ello con estas palabras:

Defecto muy frecuente en la construcción de "haver-hi" y "fer" es su falsa concordancia plural cuando la palabra que hace de complemento es plural. "Hi han persones" por "hi ha persones" (Badia, 1962: 191).

Por su parte, J. Solá $(1973 ; 1986 ; 1987)$ aporta algunos datos que pueden ayudarnos en la interpretación del fenómeno. Además de hacerse eco de las discrepancias existentes entre quienes defienden el valor del sujeto para el sintagma nominal, y por lo tanto, la concordancia, y quienes consideran ésta como un rasgo de habla "vulgar" — polémica a la que considera inútil adherirse_- el lingüista catalán construye un cuadro bastante ilustrativo de las diferencias existentes entre diversas lenguas con respecto a este problema sintáctico:

Llatí $(+)=$ Est, sunt; anglés $(+)$ : there is / there are; barceloni (+): hi ha / hi han; francés (-): il y avait des jours qu'on attendait l'armistice; castellá (-en general): había un hombre / había cinco hombres; alemany (-): Es gibt einen Tish (Badía, 1947: 17), / Es gibt zwei Tische (J. Solá 1973: 14-15)

Para lo que aquí nos interesa, observemos cómo, frente a lo visto en el español peninsular, en opinión de Solá el fenómeno de la concordancia es general, al menos para el dialecto barceloní, el de mayor influencia social, sin duda, del ámbito catalán. Y a nuestro juicio, un estudio del dialecto valenciano que se detuviera en el análisis sociolingüístico del problema, arrojaría probablemente una impresión similar a la que se señala para el catalán de Barcelona.

Por otro lado, es importante destacar que esta lengua posee más posibilidades estructurales incluso que el propio español para impulsar internamente el hecho de la concordancia. Badia (1962: 190) indica, por ejemplo, que el verbo "haver" en catalán se construye siempre con el adverbio pronominal "hi", de manera que, en rigor, hay que hablar del verbo "haver-hi", como se ve en los siguientes ejemplos:

6. A este respecto, puede resultar reveladora la lectura de cualquiera de los dos diarios publicados en Valencia, Levante y Las Provincias, en los que pueden apreciarse las diferencias entre los artículos escritos por periodistas de la comunidad y aquéllos que llegan al periódico desde fuera, bajo la forma de colaboración habitual. 
(8) Hi haura molta gent.

(9) no sé si hi pot haver cap altra solució

En castellano pervive también un recuerdo de este pronombre adverbial (heredero del ibi latino), pero solamente en una ocasión, como morfema trabado en la tercera persona singular del indicativo:

(10) Hay fiestas en ese pueblo.

(11) Hay mucha gente en la manifestación

A nuestro juicio, la explicación que buscábamos puede hallarse en el diferente tratamiento que del mencionado pronombre adverbial hacen ambas lenguas. Mientras en catalán "hi" es un morfema libre, que aparece en todas las oraciones de este tipo y siempre antepuesto al verbo-lo cual permite mucho más fácilmente la atracción del complemento-, en español, sin embargo, existe por lo menos un caso, la tercera persona del presente de indicativo-muy habitual en el habla, no lo olvidemos-en donde dicha concordancia es totalmente imposible. De esta manera, se explica la agramaticalidad de (13), pero no la de (14):

(12) *hayn fiestas en ese pueblo.

(13) hi han flors al jardí

\section{El trabajo empírico: cuestiones metodológicas}

Para poner a prueba nuestras hipótesis hemos realizado una investigación empírica en una comunidad de habla valenciana en la que valenciano ${ }^{7}$ y castellano conviven habitualmente en una situación de contacto que algunos han descrito como bilingüismo social y otros como una manifestación de diglosia y/o conflicto lingüístico (cf. Rojo 1985; Ninyoles 1969) ${ }^{8}$.

Con el fin de analizar la posible discriminación positiva de los elementos autóctonos sobre el español de esta comunidad, se han tomado como criterios fundamentales tanto la lengua materna (CASTELLANO, VALENCIANO) y habitual de los hablantes (BILVAL, BILCAST y MONOLING9), como su lugar de origen (VALENCIA, PVC y RESTO ${ }^{10}$. Finalmente, se han considerado también otras variables sociológicas habituales en los estudios sociolingüísticos:

7. Por razones estilísticas utilizaremos indistintamente las denominaciones valenciano o catalán para referirnos a la lengua autóctona hablada en las comunidades de habla valencianas.

8. Los datos proceden de una investigación sociolingüística más amplia realizada en el distrito de Campanar de la ciudad de Valencia. 
1) Sexo: HOMBRE, MUJER

2) Edad: JOVEN (15 a 25 años), MADURO (de 26 a 40 años), ADULTO (de 41 a 60 años) y ANCIANO (mayores de 60 años).

3) Nivel de estudios: P.I (Primarios incompletos), P.C.(Primarios completos), SECUND (Secundarios) y MEDSUP (Estudios universitarios medios y superiores).

4) Clase socioeconómica: BAJA, MEDIA y ALTA ${ }^{11}$

La muestra está compuesta por 196 informantes seleccionados al azar por cuotas de sexo y edad.

Por otro lado, los datos del habla han sido obtenidos a partir de la cumplimentación por los informantes de dos pruebas que describimos seguidamente ${ }^{12}$ :

$1^{\circ}$. En primer lugar, lo que hemos denominado en otro lugar (Blas 1993: 82) test de interferencias, un cuestionario compuesto por una serie de estímulos conversacionales a los que deben responder los informantes. Tras un periodo de adaptación para mitigar los efectos de la paradoja del observador (Labov 1972), entrevistador e informante llevan a cabo un experimento -sin que el interlocutor sea consciente de sus consecuencias- a través del cual el primero formula una pregunta sobre el entorno social o personal del segundo, que es respondida por éste ${ }^{13}$. Esta circunstancia exige una respuesta rápida por parte del hablante en la que se espera que aparezcan las variantes lingüísticas objeto de estudio. A este respecto, hay que subrayar que la metodología se mostró eficaz en un porcentaje alto de las entrevistas realizadas. Por otro lado, pensamos que si bien el registro de habla obtenido mediante este sistema no coincide exactamente con el estilo casual, las características del método experimental permiten que sea lo más cercano a éste que es posible obtener mediante el empleo de técnicas indirectas para el estudio de la variación sintáctica (Blas 1993; Blas y Porcar, en prensa)

9. $\mathrm{BILVAL}=$ Bilingües pero con predominio del valenciano como lengua habitual o dominante; BIL$\mathrm{CAST}=$ Bilingües con predominio del castellano; y MONOLING: hablantes que sólo utilizan activamente una lengua, en este caso, el castellano

10. VALENCIA: Nacidos y residentes en Valencia desde siempre; PVC: Nacidos en otras comarcas de habla catalana (País Valenciano y Cataluña); y Resto: inmigrantes procedentes de otras áreas españolas ajenas al área de influencia lingüística catalana.

11. Esta clasificación tripartita, de cuyos problemas, no obstante, somos conscientes, ha sido obtenida siguiendo el criterio metodológico clásico en sociolingüística de combinar diversos factores sociológicos. En este trabajo, se ha atendido a los siguientes: a) los ingresos del informante o del cabeza de familia en el caso de los más jóvenes: b) el área urbanística de residencia, y c) el nivel de estudios.

12. Sobre la dificultad de trabajar en el estudio de la variación gramatical con las mismas herramientas para la obtención de datos que en la variación fónica, nos hemos ocupado en trabajos anteriores (Blas 1993; Blas y Porcar (en prensa). En ellos hemos justificado el uso de esta metodología para estudios como el presente, en los que la variable sintáctica presenta dos variantes perfectamente discriminables e idénticas semántica y referencialmente (a este respecto, recuérdese la famosa polémica entre Labov y Lavandera).

13. Por ejemplo, a una pregunta como ¿Cuántos médicos cree que puede haber en la FE (el hospital de Valencia, situado en el distrito de Campanar)?, es bastante probable que el interlocutor responda mediante construcciones como "Habrán/Habrá...; puede que hayan/haya..., antes habían/había, etc." 
$2^{\circ}$. Un test de aceptabilidad, que trata de analizar los juicios subjetivos que los hablantes realizan acerca de esas mismas variantes lingüísticas. Esta prueba, que en nuestro caso contempla tres posibilidades -aceptable, inaceptable y dudosa-, puede servir además para evaluar la seguridad o inseguridad lingüística de los hablantes. Sin embargo, y con el fin de facilitar al lector la comprensión del análisis empírico, en la exposición de los datos nos haremos eco sólo de las frecuencias correspondientes a los juicios favorables -aceptables- y desfavorables -inaceptables o dudosas-14.

El conjunto de los datos empíricos ha sido sometido a diversos procesos estadísticos, como el recuento de frecuencias absolutas, su traducción a porcentajes sobre el global, medidas de distribución central y de dispersión, pruebas de significación probabilísticas como el estadístico $t$ - de student para la comparación de medias, así como coeficientes de correlación como el de Pearson, para determinar el grado en que las distintas variables están relacionadas entre sí.

Finalmente, hemos dividido la variable lingüística objeto de estudio en dos subvariables, con el fin de analizar la hipotética incidencia de algunas formas gramaticales en la variación sintáctica. En este sentido, hemos separado las formas simples de haber de las formas perifrásticas:

1) HABIAN1 (formas simples):

Ejemplo: En aquel cuartel habían muchos soldados.

2) HABIAN2 (formas perifrásticas)

Ejemplo: En aquel cuartel puede que hayan 500 soldados.

Deben de haber unos 500 soldados,

etc.

\section{Los datos}

\subsection{Test de interferencias}

En el cuadro $n^{\circ} 1$ el lector tiene una representación gráfica del grado de concordancia practicado en la comunidad de habla, así como su distribución por cada uno de los grupos sociales en que quedó estructurada la sociedad.

En primer lugar sobresale el hecho de que la variante que supone la concordancia es claramente mayoritaria en el conjunto de la sociedad. Por otro lado, obsérvese cómo no existen apenas diferencias cuantitativas en la realización de las dos subvaria-

14. Las respuestas al test de aceptabilidad se realizaron a partir de los juicios emitidos respecto a frases como : En aquel cuartel habían muchos soldados; Deben de haber cerca de mil alumnos en ese colegio, etc. 
bles consideradas, lo cual significa que la concordancia se aplica al mismo nivel tanto en las formas simples como en las perifrásticas $(t 1,95 ; p$. .001). La distribución social de la variante es asimismo muy similar entre los grupos que componen la comunidad, como muestra el valor muy elevado del coeficiente de correlación (.940).

Por lo que se refiere a la incidencia de las variables sociológicas, digamos en primer lugar que el sexo es el factor menos discriminante de todos. Obsérvese cómo los porcentajes de realización de la concordancia en ambas subvariables es prácticamente idéntico.

Distinto es el caso, sin embargo, cuando asistimos a la covariación entre los hechos lingüísticos y el resto de las variables sociales. Por ejemplo, el nivel de estudios y la clase socioeconómica establecen una cierta discriminación jerarquizante entre los distintos grupos. Los individuos con una formación académica escasa y los clasificados en los estratos socioeconómicos más bajos se sitúan a la cabeza de las realizaciones de la concordancia. Por el contrario, los informantes con estudios superiores y los que ocupan los estatus más altos de la sociedad sobresalen justo por lo contrario. Con todo, y a la vista de los datos empíricos, la variable nivel de estudios se revela en este caso más relevante que la clase socioeconómica. Entre estos dos extremos, los demás grupos muestran una gradación descendente, ocupando una posición intermedia, aunque eso sí, mucho más cerca del comportamiento de los grupos sociales bajos.

La edad es asimismo un factor relevante, si bien ahora las realizaciones más alejadas de la media general corresponden al grupo de hablantes de mayor edad. Éstos figuran a la cabeza en la realización de la concordancia con porcentajes que hacen casi categórica esta variante. Los demás grupos muestran un comportamiento mucho más homogéneo y a este respecto, destaca el hecho de que no sean los jóvenes quienes sobresalgan por unas realizaciones más acordes con la norma del español, como cabría esperar por su mayor vinculación con la normativa aprendida a través del sistema educativo.

Pero son, si duda, la adscripción lingüística y el origen de los hablantes las variables que ejercen un papel discriminante más decisivo. Así se desprende de las diferencias porcentuales elevadas -y significativas estadísticamente- entre los hablantes que tienen como lengua materna el castellano y los que tienen el catalán. Como puede apreciarse en el gráfico, las cifras del primer grupo doblan casi a las del segundo, es decir, que para los valencianohablantes la concordancia es prácticamente la única variante empleada (sólo un 16,6\% realiza la variante normativa, todos ellos pertenecientes además al grupo de estudios superiores), mientras que entre los castellanohablantes originarios se aprecia una mayor variabilidad.

Las diferencias son todavía más significativas cuando consideramos la lengua habitual de los hablantes. Así, los bilingües encabezan con notable diferencia sobre los demás las realizaciones concordantes, pero ello es más evidente aún en aquéllos que tienen el catalán como lengua dominante. Por el contrario, los castellanohablantes monolingües se sitúan ligeramente por debajo de la norma general, aunque hay 
que reconocer que el porcentaje de los que practican la concordancia no es nada despreciable.

A esto último contribuye el hecho de que entre los monolingües castellanohablantes se integre todo el grupo de inmigrantes llegados a esta comunidad procedentes de regiones de habla no catalana. Obsérvese cómo son precisamente estos hablantes junto con los de estudios superiores- quienes cierran la tabla de frecuencias en las dos subvariables lingüísticas consideradas. Sin embargo, la concordancia es una elección casi categórica para los originarios de comarcas de habla catalana, especialmente si proceden de zonas donde el catalán tiene una extensión social superior a la de la ciudad de Valencia. En este sentido, no es irrelevante que las diferencias porcentuales entre los grupos PVC y VALENCIA sean de casi veinte puntos, distancias que los test de probabilidad estadísticos dan como significativos para la muestra analizada.

\subsection{Test de aceptabilidad}

El primer dato que sobresale en el análisis de los datos correspondientes al test de aceptabilidad (véase cuadro $\mathrm{n}^{\mathbf{0}}$ 2) es que los niveles de aceptación de la concordancia en las oraciones con haber es con frecuencia superior al grado de realización del fenómeno del que acabamos de dar cuenta. Como veremos, este hecho es predicable tanto del conjunto de la sociedad como de la mayoría de los grupos que la componen.

Es necesario subrayar asimismo que en el presente caso existen algunas diferencias cuantitativas entre las dos subvariables lingüísticas consideradas en el trabajo. A este respecto destaca el hecho de que las formas perifrásticas son todavía más aceptadas que las correspondientes formas simples (76,33\% vs. 69,53\%, respectivamente con un estadístico $t: 5,7 p$ : .05). También son relevantes las diferencias que a este respecto se aprecian entre las respuestas proporcionadas por algunos grupos sociales cuyas discrepancias son aquí mucho mayores que las observadas en el test anterior. Véanse, por ejemplo, en los grupos de Jóvenes o entre los informantes con Estudios Secundarios.

Por otro lado, y como hemos apuntado más arriba, la mayoría de los grupos sociológicos en que estructuramos la comunidad muestra unas actitudes muy positivas hacia las variantes no normativas, actitudes que superan incluso en grado a lo que esos mismo grupos realizan cuando su conciencia lingüística no es estimulada. Ello es especialmente significativo entre los hablantes menos alejados de la norma en el test de interferencias. Compárense a este respecto las frecuencias relativas obtenidas en ambos test por grupos como Maduros, Resto y los que tienen el castellano como lengua materna y/o dominante15. El resto de la sociedad, es decir, los sectores sociales

15. En el tratamiento de los datos empíricos del test de aceptabilidad decidimos dejar fuera al grupo de Ancianos por los problemas diversos que se plantearon con sus miembros en la realización de la prueba (v. gr. algunos no comprendían el sentido del test, otros apenas sabían leer, etc.) 
que más realizan la concordancia manifiestan generalmente una actitudes todavía más positivas, pero ahora las distancias con algunos de los anteriores no son tan elevadas, de lo que se desprende una gran homogeneidad social en el enjuiciamiento de la variable lingüística. Con todo, los hablantes con estudios superiores y los de clase social elevada muestran unos juicios subjetivos mucho más negativos hacia las variantes no normativas.

\section{Discusión}

Los datos anteriores confirman nuestra hipótesis inicial de que la concordancia en oraciones con haber es una variante muy extendida en la sociedad valenciana y además muy positivamente valorada por la mayoría de la comunidad. Por otro lado, el fenómeno se halla plenamente consolidado, como lo demuestra el grado altísimo de realizaciones concordantes por parte de los hablantes más adultos. De hacer caso a la teoría laboviana acerca de la pervivencia de los principales rasgos del repertorio lingüístico de los hablantes a lo largo de su historia personal (Labov 1972), habría que colegir necesariamente que el fenómeno es antiguo en nuestra comunidad y que no nos encontramos necesariamente ante un estadio expansivo del mismo, como ocurre en otras comunidades de habla peninsulares (recuérdese lo escrito más arriba en el apartado 3).

Ahora bien, no toda la comunidad de habla participa del fenómeno en la misma medida, y en este sentido las variables de adscripción sociolingüística y el origen de los hablantes se revelan como las más decisivas en la variación. El hecho de que los valencianohablantes habituales o con el catalán como lengua materna realicen la concordancia y manifiesten unas actitudes muy positivas hacia la variante en un grado superior a los otros grupos parece confirmar asimismo la tesis central de este trabajo, es decir, que el fenómeno puede responder en nuestras comunidades de hablas a un caso de convergencia gramatical entre las lenguas implicadas en la sociedad y para el que, aparte de los factores internos evidentes ya reseñados, la influencia del catalán resulta decisiva.

Por otro lado, estos datos confirman las hipótesis formuladas en alguna ocasión acerca de la relación entre el grado de competencia lingüística de los hablantes y el nivel de interferencia en el uso de la segunda lengua (Andersen 1982). Como se ha visto en el apartado anterior, la frecuencia en la realización de la concordancia aumenta conforme lo hace también el grado de restricción en el uso del español por parte de los hablantes. Así, tanto los bilingües que tienen el catalán como lengua más habitual como los nacidos en comarcas del área lingüística catalana, en las que el uso social e individual de la lengua autóctona es superior al que se da en la ciudad de Valencia, el nivel de convergencia gramatical es también claramente superior ${ }^{16}$.

16. En ese sentido, nuestros resultados no coinciden con los hallados por Mougeon y Beniak (1991) en el estado de Ontario (Canadá), a propósito de algunas variables lingüísticas de la segunda lengua. En 
Ahora bien, la presencia del fenómeno incluso entre los sectores de la sociedad menos "autóctonos", en niveles que, aunque claramente inferiores al resto, cabe calificar como notables, exigen una matización de las conclusiones anteriores. A este respecto, el cuadro sociolingüístico que ofrece nuestro fenómeno en la sociedad valenciana pone en duda los mecanismos del cambio lingüístico de inspiración laboviana. Como es sabido, el modelo descrito por Labov (1972) para describir los cambios en comunidades monolingües implica la introducción de una innovación por un individuo o un grupo pequeño de hablantes pertenecientes a una determinada clase social, la subsiguiente extensión del mismo entre los demás componentes de ese grupo y su eventual adopción posterior por el resto de la sociedad. Por el contrario, nuestra investigación sugiere un perfil variacionista diferente. El fenómeno se halla tan extendido y aceptado por la sociedad que hace difícil pensar en un mecanismo de propagación como el descrito. Como han indicado Mougeon y Beniak (1991: 14), en las situaciones de contacto intensas y que permanecen en el tiempo tras muchas generaciones, las innovaciones y su ulterior expansión pueden surgir con independencia y simultáneamente en amplios sectores de la sociedad, sin que en el desarrollo del proceso la clase social tenga una relevancia como la que supone Labov. Para estos autores, la explicación de esta regla es clara: "bilingualization and restriction are overriding sociological processes that cut across the whole social spectrum and free the structural (intraand/or intersystemic) forces at play"17

En nuestro caso, las lineas generales de este modelo se confirman. El éxito social de la concordancia en la sociedad valenciana -y en otras del área lingüística catalanasugiere que estamos ante un fenómeno que previsiblemente no ha sido introducido por individuos o grupos pequeños en su origen sino que, como consecuencia del extenso bilingüismo al que hacíamos referencia, ha debido ser una constante en el uso del español por parte de extensos sectores de la sociedad. De hecho, el mecanismo laboviano del cambio, según el cual siempre se suceden dos momentos en el cambio, la innovación y su posterior propagación, puede que tampoco se haya producido en el fenómeno que nos ocupa.

La confirmación de que el cambio no ha podido surgir de un único subgrupo social, especialmente de las clases bajas, se ve reflejada también en los niveles frecuenciales alcanzados por los diferentes grupos socioeconómicos. Recuérdese que el fenómeno de la concordancia está extraordinariamente extendido por amplias capas

aquél caso, los datos obtenidos no permitían establecer diferencias entre el grado de restricción en el uso de una lengua y el nivel de interferencia.

17. De hecho el propio Labov (1972: 277-8), tras formular su hipótesis general para dar cuenta de los mecanismos del cambio lingüístico, no negaba enteramente la posibilidad de innovaciones autónomas e independientes a cargo de diferentes hablantes o pequeños grupos sociales: "We do not rule out the possibility of independent simultaneous innovation by a number of speakers; but we do find absurd the notion that an entire community would change simultaneously without reference to each other, without a gradual transfer of the pattern from speaker to speaker" 
sociales, que incluyen tanto a los niveles bajos como a los medios, grupos que conjuntamente representan a la inmensa mayoría de la comunidad. Sólo los representantes de las clases altas se salen de esta norma, aunque sus niveles de realización de la concordancia no sean tampoco nada desdeñables. Por otro lado, el comportamiento de este grupo social se ve influido por otro factor que hemos considerado en la investigación, el nivel de estudios, y por tanto, el grado en que la prescripción gramatical puede afectar a la actuación lingüística de los hablantes, así como a sus actitudes. Ello permite explicar mejor el comportamiento discordante del grupo social de clase alta, ya que la mayor parte de sus componentes pertenecen también al nivel de estudios superiores, nivel que alcanzó el grado más bajo de realización de la concordancia, así como también el mayor rechazo hacia la variante autóctona. Por otro lado, tanto el comportamiento de estos hablantes como la escala jerárquica descendente -de mayor a menor grado de interferencia- que dibujan los otros grupos de instrucción son indicios más que suficientes para confirmar la importancia de la prescripción gramatical como factor explicativo en la variación sociolingüística.

\section{Conclusiones}

A lo largo de las página anteriores esperamos haber demostrado que la extraordinaria extensión social de la concordancia entre haber y el sustantivo en construcciones existenciales no puede explicarse sin acudir a la influencia del catalán en las comunidades de habla del área linguística catalana . Esta lengua, que convive con el español desde hace siglos en estas áreas geográficas, posee una estructura sintáctica semejante que, si bien tampoco es aceptada por la norma, resulta en la práctica una variante de elección -cuasi- categórica en la sociedad. Tanto una lengua como otra tienen suficientes razones estructurales, puramente internas, para explicar el origen de la innovación gramatical, ya que la impersonalidad -y la no concordancia- constituye una anomalía que rompe con el paradigma de la concordancia entre sujeto y verbo, con repercusiones en la morfología verbal. Pero la explicación del origen a partir de mecanismos internos de la propia lengua no implica por si sola la propagación y el éxito del cambio en la sociedad. Por el contrario, en ocasiones éstos últimos vienen impulsados por motivos ajenos a la propia lengua, como ocurre en las situaciones de contacto lingüístico en las que las lenguas tienden a neutralizar sus diferencias lingüísticas. En ese sentido, hemos defendido la consideración del fenómeno como un caso de convergencia sintáctica en cuyo origen y difusión social intervienen factores estructurales y no estructurales y entre éstos, la interferencia lingüística como el más importante.

Contrariamente a la opinión de aquellos lingüistas que advierten sobre la necesidad de acudir a la influencia interlingüística como factor explicativo tan sólo cuando no puede hallarse una explicación al cambio por medio de los hechos inherentes a la propia lengua, consideramos que la posibilidad de la causación múltiple no puede ser 
descartada nunca de antemano. Y para ello, un conocimiento etnográfico y sociolingüístico lo más completo posible de la sociedad es indispensable. Como han recordado Thomason y Kaufman (1988: cap. 3.3), las explicaciones sobre el cambio lingüístico y sobre la variación en general, deben ser lo más completas posible, huyendo así de los reduccionismos a que conducen las explicaciones simplistas, por muy atractivas que puedan parecer a simple vista. Y este reproche -nos parece- vale tanto para aquéllos que han visto de una forma irreflexiva en la interferencia lingüística la causa de cualquier innovación, cuanto para los que, bajo supuestos cientifistas, siguen considerando ésta como un ente autónomo, que rige sus destinos a partir de mecanismos puramente internos y ajenos, por tanto, a cualquier influencia externa.

Por ello, el hecho de que el fenómeno que nos ocupa haya sido documentado en otras áreas del español, incluso con gran éxito social también, creemos que no invalida nuestras hipótesis. Antes al contrario, nos reafirma en la idea de que la causa de un mismo hecho estructural puede ser explicada en ocasiones con el auxilio exclusivo de factores internos, mientras que en otras puede hacerse también con el concurso de motivos externos, especialmente en aquellas comunidades de habla bilingües en las que la otra lengua presenta una estructura similar.

Por último, este trabajo pretendía contribuir, siquiera mínimamente, a cubrir el enorme vacío existente en torno a la bidireccionalidad de la interferencia. La situación de contacto catalán-español, en la que tradicionalmente la segunda lengua ha ocupado una posición social elevada en relación a la primera, ha dado lugar a lo largo de los siglos a numerosos hechos interferenciales, pero no sólo en la dirección esperada, esto es, aquélla en la que la lengua que ocupa un lugar de privilegio es la que deja su huella en la lengua baja. Es posible que estudios empíricos que, por desgracia faltan, demostraran la preeminencia de esta dirección, pero ello no destierra la impronta dejada en el español por la dirección contraria. Cualquiera que conozca con detalle el español hablado en las comunidades de habla del área lingüística catalana sabe que está salpicado aquí y allá por rasgos procedentes del catalán, unos plenamente consolidados en la sociedad -integrados-, junto a otros que ocurren sólo ocasionalmente en el habla espontánea de los hablantes, generalmente bilingües, pero no necesariamente. En ese sentido, el grado de convergencia gramatical al que español y catalán han llegado en algunos paradigmas sintácticos es sumamente interesante.

\section{Bibliografía}

ALCINA, J. y BLECUA, J. M. (1975): Gramática española, Barcelona, Ariel.

ANDERSEN, R.W. (1982): "Determining the Linguistic Attributes of Language Attrition”, en Richard D. Lambert y Bárbara F. Freed (eds.): The Loss of Language Skills, Rowley, Mass. Newbury House Publishers, 83-118.

BADIA, A. M. (1962): Gramática Catalana, Madrid, Gredos. 
BELLO A. (1945): Gramática de la lengua castellana, Sopena, Buenos Aires.

(1847): Gramática de la lengua castellana destinada al uso de los americanos,

Santiago de Chile. Nueva edición, con notas de R. José Cuervo, Bogotá, 1874.

BENTIVOGLIO, P. y SEDANO, M. (1992): "El espanol hablado en Venezuela”, en Hernández, C.(ed.) (1992), 775-802.

BLAS, J.L. (1991): "Problemas teóricos en el estudio de la interferencia lingüística", Revista Española de Lingüística, 21.2, págs. 265-289.

(1993): La interferencia lingüística en Valencia (dirección catalán—> español ). Estudio sociolingüístico, Castellón, Universidad Jaume I.

(1994): "La comunidad de habla. Caracterización sociolingüística del concepto y aplicaciones posibles para la descripción del bilingüismo peninsular", The Bilingual Review 19.1, págs. 9-24.

BLAS, J.L. y PORCAR, M. (en prensa): "El empleo de las formas -ra y -se en las comunidades de habla castellonenses. Aproximación sociolingüística”, Español Actual .

CARAVEDO, R. (1992): "Espacio geográfico y modalidades linguísticas en el espanol del Perú", en Hernández, C.(ed.) (1992), 719-742.

COROMINAS, J. (1954): Diccionario crítico-etimológico de la lengua castellana. Madrid, Gredos.

DE GRANDA, G. (1991): “De nuevo sobre la causación múltiple en el español de América (a propósito de dos rasgos morfosintácticos del español paraguayo)", en G. de Granda ed.: El español en tres mundos. Retenciones y contactos lingüísticos en América y África, Valladolid, Junta de Castella y León, 41-54.

EMENEAU, M. B. (1962/80): "Bilingualism and structural borrowing”, en Anwar Dil (ed.): Language and Linguistic Area, Stanford, Standford University Press, 85-104.

FERNANDEZ RAMIREZ, S. (1986): Gramática española. Volumen IV: El verbo y la oración, Arco/Libros, Madrid.

GARCIA YEBRA, V. (1983): “¿Complemento directo o sujeto con las formas unipersonales de haber?" RFE, LXIII, 33-71.

GILI GAYA, S. (1961): Curso Superior de sintaxis española. Biblograf, Barcelona (13 ${ }^{\mathrm{a}}$ ed., 1981).

GUMPERZ, J. y WILSON, R. (1971): “Convergence and Creolization: A Case from the Indo-Aryan/Dravidian Border”, en Del Hymes (ed): Pidginization and Creolization of Languages, Cambridge, Cambridge University Press, 151-67.

HERNANDEZ, C.(ed.) (1992): Historia y presente del Español de América, Valladolid, Junta de Castilla y León/Pabecal.

JAKOBSON , R. (1938/62): "Sur la théorie des affinités phonologiques entre des langues”, en Selected writings, vol. I, 234-46. The Hague, Mouton.

KANY, Ch. (1945): American-Spanish Syntax, Chicago, University of Chicago Press.

LABOV, W. (1972): Sociolinguistic Patterns, Philadelphia, University of Pennsylvania Press. 
MACKEY, W.F. (1976): Bilinguisme et contact des langues, Paris, Klincsieck.

MENDOZA, José G (1992): “Aspectos del castellano hablado en Bolivia”, en C. Hernández (ed.) (1992), 437-500.

MONTES, J. J. (1992): El español hablado en Colombia, en C. Hernández (ed.) (1992), 519-542.

MOUGEON, R y BENIAK, E. (1991): Linguistic Consequences of Lanuage Contact and Restriction. The case of French in Ontario, Canada. Oxford, Clarendon Press.

MORENO CABRERA, J.C (1991): Curso de Lingüística general, Madrid, Síntesis.

MORENO DE ALBA, J.G. (1992): "El español hablado en México", en C. Hernández (ed.) (1992), 627-648.

NINYOLES, R. (1969): Conflicte lingüístic valencià, València, Ed. Tres i Quatre.

PAYRATO, LL. (1985): La interferència lingüística (Comentaris i exemples catalácastellá), Barcelona, Ed. Curial-Publicacions de l'Abadia de Montserrat.

ELIAS-OLIVARES (ed.): Spanish in the US Setting:Beyond the Southwest, Washington, DC, National Clearinghouse for Bilingual Education, 108-29.

QUILIS, A. (1992): "Rasgos generales sobre la lengua española en el Ecuador”, C. Hernández (ed.) (1992),593-606.

R.A. E. (1931): Gramática de la lengua castellana, Madrid, Espasa-Calpe.

ROJO, G. (1985): "Diglosia y tipos de diglosia", en Philologica hispaniense in honorem M. Alvar, II, Madrid, Gredos, 603-17.

SALVÀ, V. (1830): Gramática de la lengua castellana según ahora se habla. París.

SECO, M. (1961/86): Diccionario de dudas y dificultades de la lengua espanola. Madrid, Espasa-Calpe.

SILVA-CORVALAN, C. (1986): "Bilingualism and Language Change: The extension of estar in Los Angeles Spanish", Language, 62/3, 587-608.

(1994): Language contact and change, Oxford, Oxford U. P,

(1995): "The Study of Language Contact: An Overwviw of the Issues", en C.

Silva Corvalán (ed.): Spanish in Four Continents. Studies in Language Contact and Bilingualism. Washington, D.C., Georgetown University Press, 3-14.

SOLÀ, J (1973): Estudis de sintaxi catalana I y 11, Barcelona, Edicions 62. (1986): Gramàtica catalana, Barcelona, Publicacions de la Abadia de Montserrat.

(1987): Questions controvertides de sintaxi catalana, Barcelona, Edicions 62.

THOMASON, S.G. y KAUFMAN, T. (1988): Language Contact, Creolization and Genetic Linguistics, Berkeley, University Of California Press.

TRUDGILL, O. (1983): On Dialect, Oxford, Basil Blackwell.

VOGT, H. (1954): "Language Contacts", Word, 10, 365-74.

WEINREICH, U. (1953): Languages in contact, The Hague, Mouton.Trad. cast: Lenguas en contacto. Descubrimientos y Problemas. Ediciones de la Biblioteca de la Universidad Central, Caracas, 1974. 
A PROPÓSITO DE UN CASO DE CONVERGENCIA GRAMATICAL

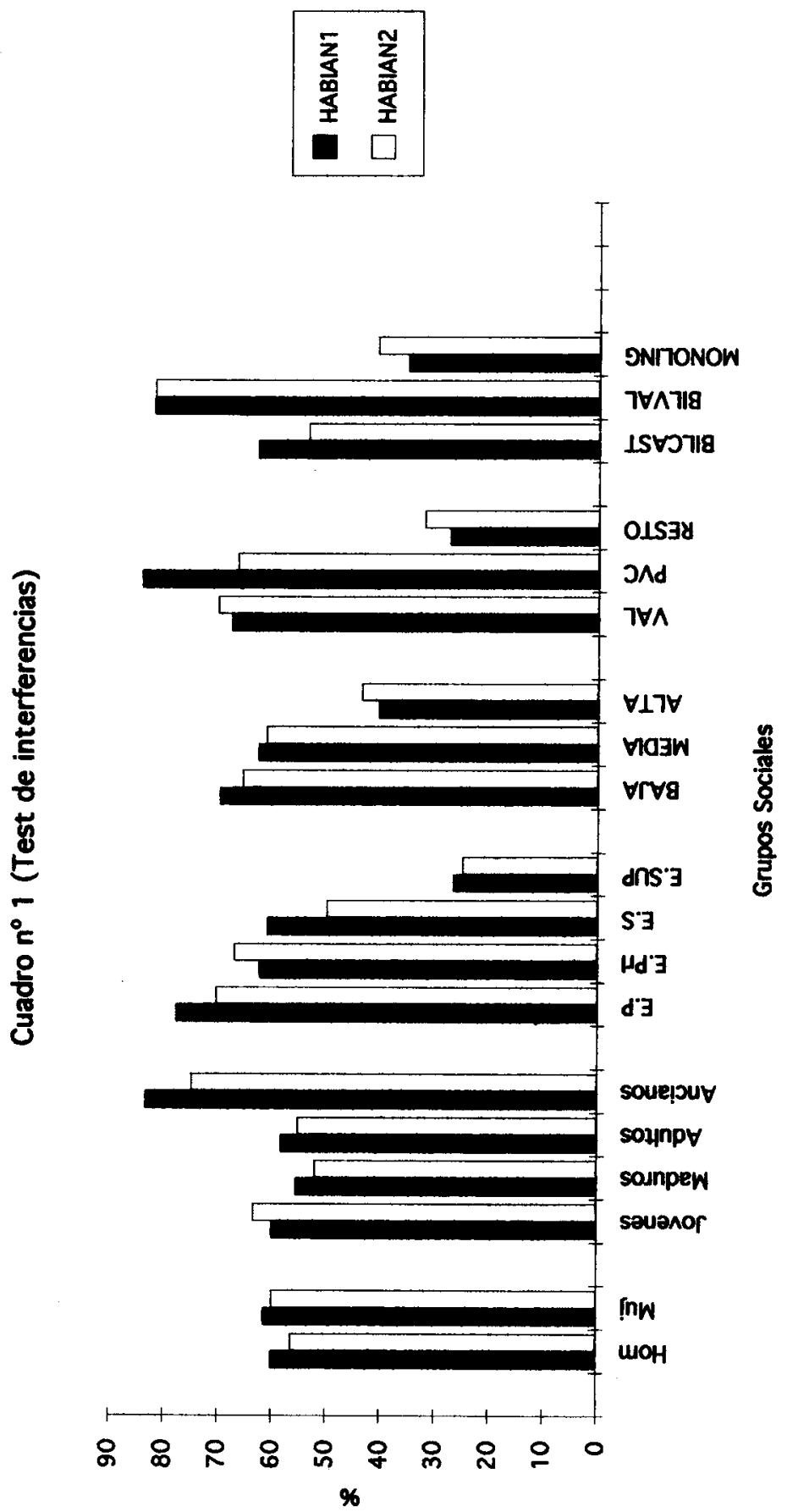


JOSÉ LUIS BLAS ARROYO

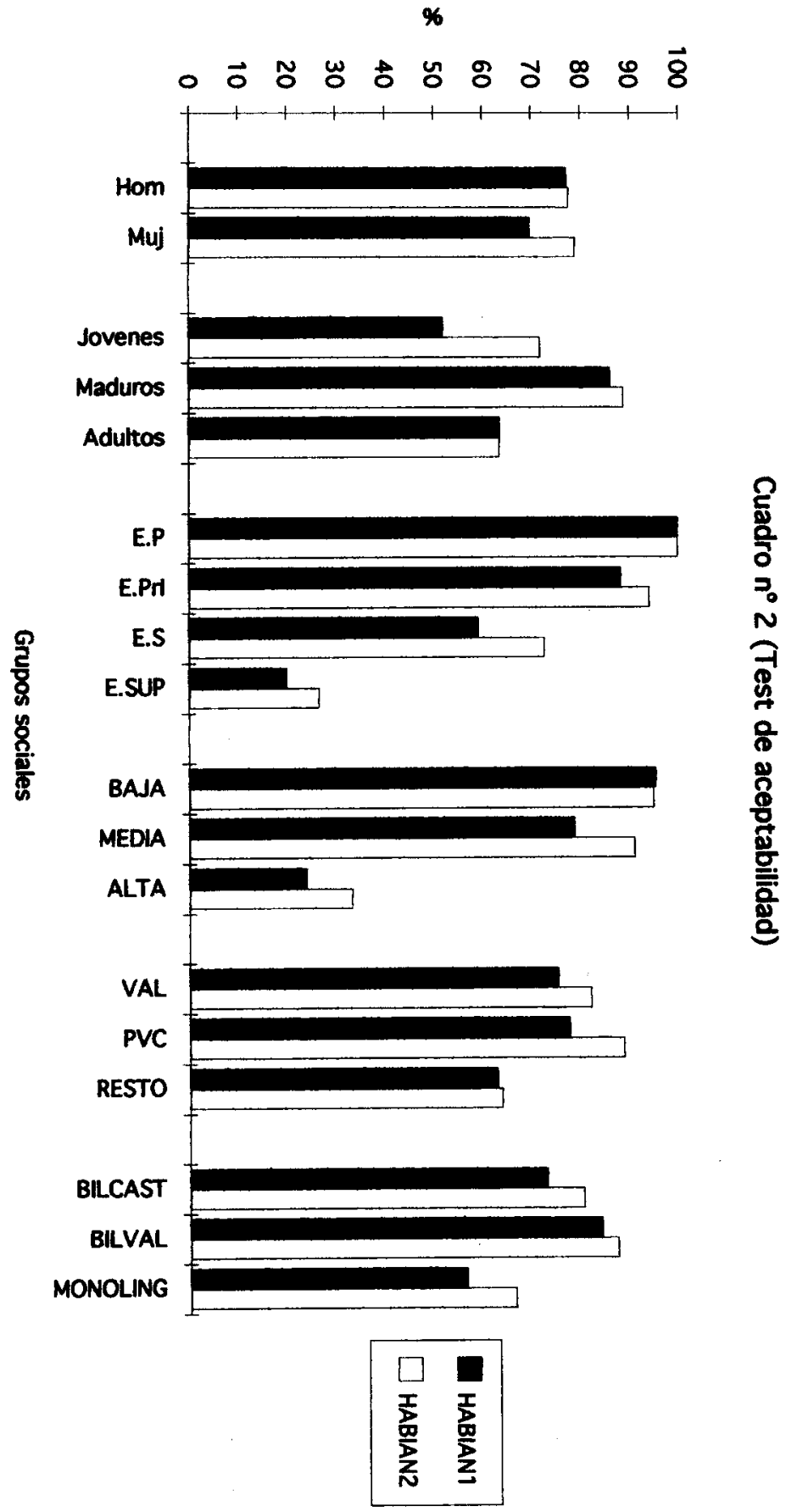

